\title{
Prospects of Aloe vera and its Bioactive Compounds in Diabetes: Critical Review
}

\author{
Mukesh Kumar Sharma ${ }^{1,2 *}$ (D), Jagat Chauhan ${ }^{1}$, Mohan Kumar ${ }^{1}$, \\ Chetan Kumar Joshi ${ }^{3}$, Sandeep Sharma ${ }^{4}$ and Chhagan Lal ${ }^{5}$ \\ ${ }^{1}$ Department of Biotechnology, Maharaj Vinayak Global University, Jaipur, Rajasthan, India. \\ ${ }^{2}$ Department of Botany, Vishwa Bharti PG College, Sikar, Rajasthan, India. \\ ${ }^{3}$ Department of Zoology, Government Science College, Sikar, Rajasthan, India. \\ ${ }^{4}$ Department of Physics, Government P.G. College, Jhunjhunu, Rajasthan, India. \\ ${ }^{5}$ Department of Physics, University of Rajasthan, Jaipur, Rajasthan, India.
}

\begin{abstract}
Diabetes is a significant public health issue. The global diabetes epidemic has had a tremendous impact on India, and the disease burden has increased dramatically. Diabetes is quickly increasing in prevalence, especially in Indian cities, according to data. Therefore, an ideal drug is sought that has better safety and tolerability and the most effective control of diabetes. Many effective medications come from plant sources. Natural products like onion and garlic can effectively control diabetes. In this review, we should pay attention to Aloe vera and its bioactive compounds, that with the development of traditional medicine, Aloe vera can be used to treat various diseases. Some reports have questioned the safety and efficacy of Aloe vera or its compounds, especially at different doses, and some studies have shown no side effects. In this review we also focus on benefits on human health so that Aloe vera is part of the daily diet in many countries and appears to be non-toxic, it is necessary to investigate whether aloe vera dietary supplement can be a beneficial preventive or nutritional mitigation strategy to reduce the effects of diabetes. This review focuses on Aloe vera and its biologically active compounds that play a role in the treatment or prevention of this morbid disease: diabetes, including its underlying mechanism of blood sugar lowering properties, and herbal products that have been marketed for the treatment of diabetes or the therapeutic effect of diabetes.
\end{abstract}

Keywords: Aloe vera, Bioactive compounds, diabetes, natural products, herbal medicine

*Correspondence: mukeshsharma.dt@gmail.com

(Received: July 28, 2021; accepted: November 03, 2021)

Citation: Sharma MK, Chauhan J, Kumar M, Joshi CK, Sharma S, Lal C. Prospects of Aloe vera and its Bioactive Compounds in Diabetes: Critical Review. J Pure Appl Microbiol. 2021;15(4):1781-1797. doi: 10.22207/JPAM.15.4.54

(C) The Author(s) 2021. Open Access. This article is distributed under the terms of the Creative Commons Attribution 4.0 International License which permits unrestricted use, sharing, distribution, and reproduction in any medium, provided you give appropriate credit to the original author(s) and the source, provide a link to the Creative Commons license, and indicate if changes were made. 


\section{INTRODUCTION}

More recently, studies published, the rates of hypoglycemia were higher in adolescents than in adults. The second most prevalent chronic condition is type 1 diabetes (T1D)among adolescents. Diabetes-related morbidity and premature death are the main sources of pain ${ }^{1}$ and medical expenses. Diabetes affects about nine percent of the population in the United States and costs up to $\$ 174$ billion per year. ${ }^{2}$ Effective therapy is available, but you must strike a balance between your insulin dose, nutrition, and exercise, as well as receive performance feedback on your blood glucose control levels. Therefore, even for the most determined teens, it is a problem to always apply and adhere to such a sophisticated and rigorous treatment plan. The spontaneity, longevity, and exceptionality that are characteristic of youth go against the effective management of diabetes increased attention to diabetes control, on the other hand, may aid with blood sugar control, ${ }^{3}$ and lower haemoglobin A1c (HbA1c) concentrations, in turn, may reduce the risk of diabetes complications. ${ }^{1}$ Compliance is further hindered by the painful acupuncture needed to manage sugar, the inconvenience of carrying or using a blood glucose device, and the injection of insulin, in addition to the complexity of therapy and periodic disruptions in the lives of adolescents. Although technological advances have promoted the administration of insulin injection pens or pumps, adherence to diabetes treatment programs is often problematic for patients of all ages, but it is more difficult for adolescents. ${ }^{4}$ It is difficult for adolescents to achieve and maintain the required blood glucose control because of the many factors involved in the signaling pathway (Fig. 1). And physiological changes lead to an increase in insulin resistance during adolescence. ${ }^{5}$ Additionally, as providers strengthen programs to improve blood sugar control for better results, but inadvertently increase the burden and reduce health-promoting behaviors, compliance may become more difficult. 6,7 In this review, we will focus on the natural treatment of diabetes, because convective drugs have many side effects in other parts of the organ, especially in liver metabolism, discussed recent attempts to solve these problems through promising work in this field.

\section{Focus on Epidemiology}

New in 2020, this National Diabetes

Statistics Report features trends in prevalence and incidence estimates over time. The estimates in this review (unless otherwise noted) were derived from various data systems of CDC, Indian Health Service (IHS), Agency for Healthcare Research and Quality (AHRQ), and US Census Bureau, and from published research studies. Estimated percentages and total number of people with diabetes and prediabetes were derived from the National Health and Nutrition Examination Survey (NHANES), National Health Interview Survey (NHIS), IHS National Data Warehouse (NDW), Behavioral Risk Factor Surveillance System (BRFSS), United States Diabetes Surveillance System (USDSS), and US resident population estimates. Diagnosed diabetes status was determined from self-reported information provided by survey respondents; for American Indians and Alaska Natives who accessed IHS or tribal health facilities that submitted medical records data to the IHS NDW, clinical diagnostic codes were also used. Undiagnosed diabetes was determined by measured fasting plasma glucose or $\mathrm{A} 1 \mathrm{C}$ levels. Numbers and rates for acute and long-term complications of diabetes were derived from the National Inpatient Sample (NIS) and National Emergency Department Sample (NEDS), as well as NHIS. For some measures, estimates were not available for certain racial and ethnic subgroups due to small sample sizes. An alpha level of 0.05 was used when determining statistically significant differences between groups. Age-adjusted estimates were calculated among adults aged 18 years or older by the direct method to the 2000 US Census standard population, using age groups $18-44,45-64$, and 65 years or older. Most estimates of diabetes in this report do not differentiate between type 1 and type 2 diabetes. However, as type 2 diabetes accounts for $90 \%$ to $95 \%$ of all diabetes cases, the data presented here are more likely to be characteristic of type 2 diabetes, except as noted. (https://www.cdc.gov/ diabetes/pdfs/data/statistics/national-diabetesstatistics-report.pdf)

In a meta-analysis of 603 children from diverse academic institutions in India, ${ }^{8}$ type 1 diabetes was the most common epigenetic regulation ( $89 \%)$. Other causes are rare, such 
as type 2 diabetes (which affects $6 \%$ of the population) and fibrotic pancreatic diabetes (which affects $2 \%$ of the population). Unfortunately, because population-based diabetes registries have yet to be developed, the prevalence of type 1 diabetes throughout the region has not been fully investigated. Type 1 diabetes is commonly thought to be rare among South Asians, however the report provides varying numbers. In a 1991 survey of 10,513 children in Chennai, South India, there were no known or newly diagnosed cases of Type 1 diabetes. Two more studies, one in Karachi and the other in Chennai, Pakistan, come up with completely different results. Among a 1991 study, the prevalence of Type 1 diabetes in children aged 0 to 16 years was 1.02 per 100,000 person-years. ${ }^{9}$ The 1996 Chennai study, on the other hand, found a higher incidence rate (10.5 per 100,000 person-years). ${ }^{10}$ The incidence of type 1 diabetes among South Asians and other ethnic groups was compared in a National survey. Between 1989 and 1998, a study in Leicestershire found that the number of People with diabetes among South Asians was equivalent to that of europeans and other groups (per 100,000 person / year: 19.2 vs 17.7 for women; 20, 3 vs 17.7 for males).${ }^{11}$ However, among children under 15 years studied in Yorkshire from 1978 to 2007, ${ }^{12}$ the incidence of Type 1 diabetes among non-South Asians (21.5 per 100,000 per year) was greater than that of South Asians (14.7 per 100,000 per year) in children younger than 15 years. In order to acquire trustworthy information to guide national policy against the disease, India should establish a population-based adolescent diabetes record.

\section{Focus on Genetics}

Many aspects of Type 1 diabetes' natural history and aetiology have been covered in depth elsewhere in this collection, including genetics. In conclusion, despite the high effect of hereditary variables, Type 1 diabetes is a complicated multifactorial illness that does not follow a straightforward inheritance pattern. ${ }^{13}$ The importance of the above for genetic and environmental risk factors in Type 1 diabetes is supported by early family and twin cluster investigations. ${ }^{22}$ We will continue to acquire new insights into the association between genetic risk

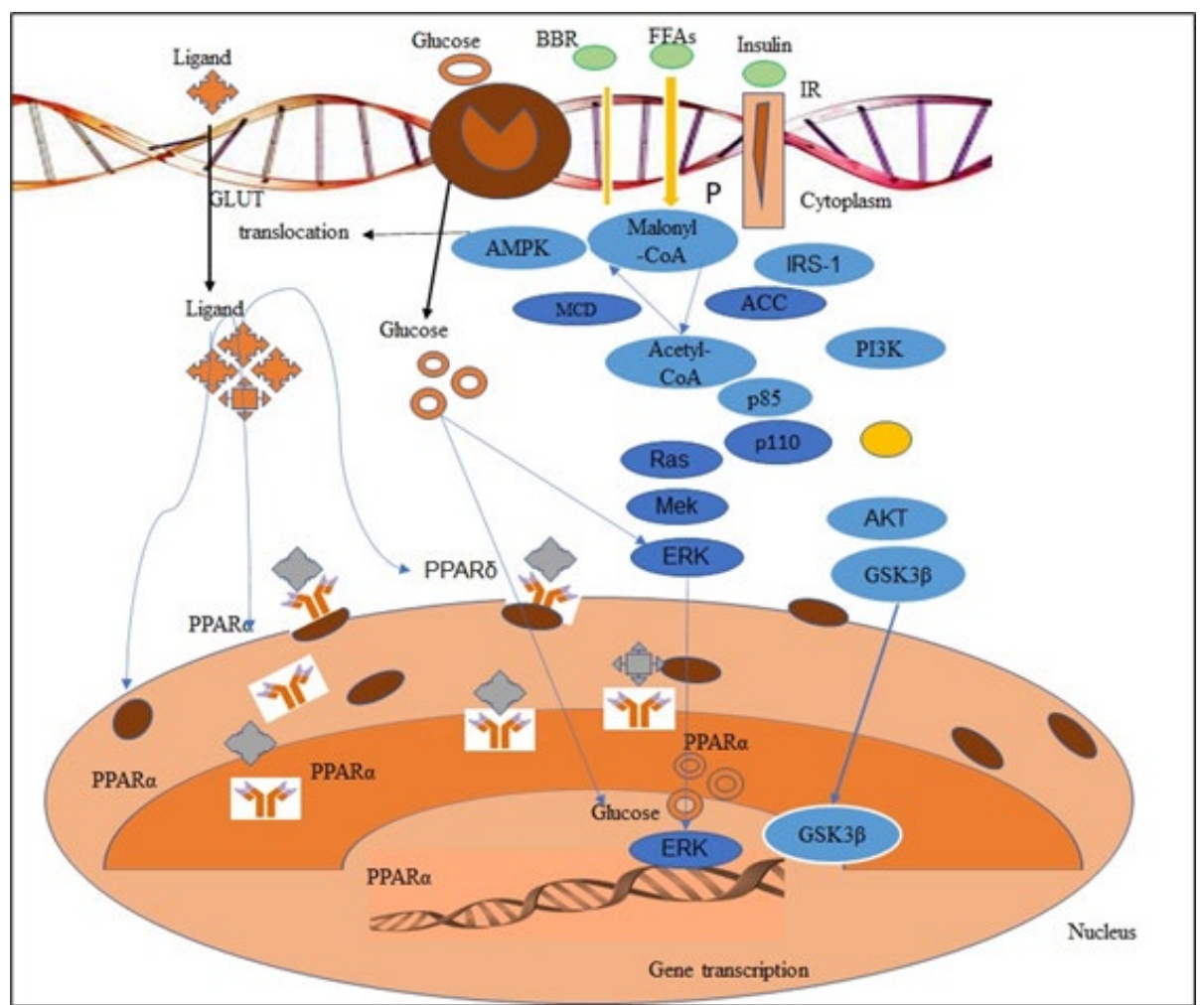

Fig. 1. Signaling Pathway participate in the diabetes. 
and autoimmune diseases that finally leads to the creation of Type 1 diabetes as our understanding of the importance of susceptibility and genes resistance increases.

\section{Prevalence of diabetes mellitus in india}

Although $95 \%$ or more cases are T2DM in India, the absolute number of patients with T1DM is important because the base of the population is great. On average, the T1DM starting age is 57 years old. This is an important period for mental and social development. Children are trying to adapt to strict daily schedules and school life, but young parents struggle to establish their own occupation career. T1DM Strict monitoring and treatment plans require considerable changes in parents' lifestyle habits and young patients. ${ }^{14}$

Today, it was to treat chronic diseases, such as diabetes. Diabetes characterized by hyperglycemia that causes alteration of lipids, carbohydrates and protein metabolism. ${ }^{15} \mathrm{An}$ ocular system. Many plants contain different natural antioxidants, tannins, flavonoids, alkaloids, vitamins $C$ and $E$, having the ability to reduce blood glucose levels. ${ }^{16}$

Medical plants are safe and safe compared to synthetic agents and are effective for the therapeutic treatment of diabetes. ${ }^{17}$

Aloe vera (Aloe) is well-known for its miraculous powers, which include pharmaceutical, health, beauty, and skin care. It's found in beverages, body lotions, cosmetics, and ointments, as well as in gel form for minor burns and sunburns. This plant, which is native to the Caribbean, South Africa, and Latin America, has a transparent gel in its leaves that is often used in cosmetic products. https://www.diabetes.co.uk/natural-therapies/ Aloe-vera.html.Aloe latex, a bitter liquid generated from the leaves' epidermis, is used in juice beverages, pills, capsules, and dental care products including toothpaste and gargle. ${ }^{18}$

The Aloe species is thought to be the most physiologically active (World Health Organization 1999). Vitamins, minerals, carbohydrates, amino acids, anthraquinones, enzymes, lignin, saponins, and salicylic acid are among the more than 75 probable active components revealed. The bitter taste and laxative effect of the leaves' exudate are thought to be due to anthraquinones, particularly babaloin. ${ }^{19,20}$ Barbaloin is a polyphenol compound that can act as an antioxidant and is responsible for free radical-mediated cytotoxicity and lipid peroxidation. ${ }^{21}$ Carotenoids, steroids, terpenes, and phytosterols have also been found to be the cause of color and fragrance. ${ }^{22}$ Aloe gel is rich in polysaccharides, including acetylmannan. The number of other potentially active compounds found in plants is responsible for many biological activities. ${ }^{18,23} \mathrm{~A}$ kind of. Vera has a variety of medicinal properties, such as anti-tumor, antiarthritis, anti-rheumatic, anti-cancer, and antidiabetic. Additionally, A. vera has been promoted to treat constipation, gastrointestinal diseases, and immune system deficiencies. ${ }^{24}$

In Latin America ${ }^{25}$ Arabian Peninsula ${ }^{26}$ and many other countries, Aloe has been used as a traditional medicine for the treatment of diabetes (DM). Previous experiments in humans and animals have shown that Aloe can reduce chronic hyperglycemia and confuse blood lipids, prevent diabetes, and also help prevent cardiovascular complications, which are the main risk factors for disease. According to reports, long-term intake of 100 grams of $A$ loe gel and 20 grams of psyllium husk has the effect of lowering blood sugar and blood lipids. The study was conducted in 5,000 patients with atherosclerotic heart disease between the ages of 35 and 65 . This population included 3167 non-insulin-dependent diabetic patients, among which decreased serum cholesterol, triglycerides, and total lipid levels and high observed Density lipoprotein cholesterol (HDL), and another 177 diabetic patients with normal blood glucose levels after fasting meals showed the need to stop all oral hypoglycemic drugs at the end of 2 months of treatment.

The effects of utilising gel of alovera alone on diabetes patients' blood glucose and blood lipid parameters have also been documented. Two clinical trials have been conducted and 72 diabetic women took just one tablespoon of Aloe gel or placebo for 6 weeks. Blood glucose and serum triglyceride profiles were significantly reduced, while cholesterol concentration was not affected. ${ }^{27}$ In the second trial, patients took either Aloe gel or a placebo combined with glibenclamide and found that 4,444 people in the Aloe group had significantly lower blood glucose and serum triglyceride concentrations. ${ }^{28}$ In another case study, in five patients with non-insulin-dependent diabetes mellitus, Aloe latex showed lower fasting 
Sharma et al. | J Pure Appl Microbiol | 15(4):1781-1797 | December 2021 | https://doi.org/10.22207/JPAM.15.4.54

Table 1. Structure and chemical constituents

\begin{tabular}{|c|c|c|c|}
\hline No. & Compound Name & PubChem ID & Structure \\
\hline 1 & Acetic acid & CID176 & \\
\hline 2 & Catechol & CID289 & \\
\hline 3 & Citric acid & CID311 & \\
\hline 4 & Gallic acid & CID370 & \\
\hline 5 & Lactic acid & CID612 & \\
\hline 6 & 3,4-Dihydrocoumarin & CID660 & \\
\hline 7 & Oxalic acid & CID971 & \\
\hline 9 & Isocitric acid & CID1198 & \\
\hline 10 & P-cresol & CID2879 & \\
\hline 11 & Emodin & CID3220 & \\
\hline 12 & 2,5-Dihydro benzoic acid & CID3469 & \\
\hline
\end{tabular}


13

Salicyladehyde

14

15

16

17

18

19

20

Anthranol

21

22

Syringic acid

Aloin B
CID14989

CID6998

CID8468

CID9064

CID10168

CID10207

CID10208

CID10639

CID10731

CID10742
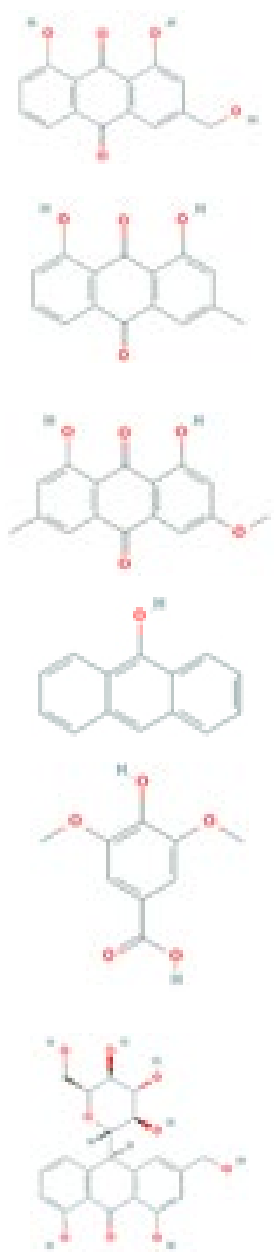
CID160190
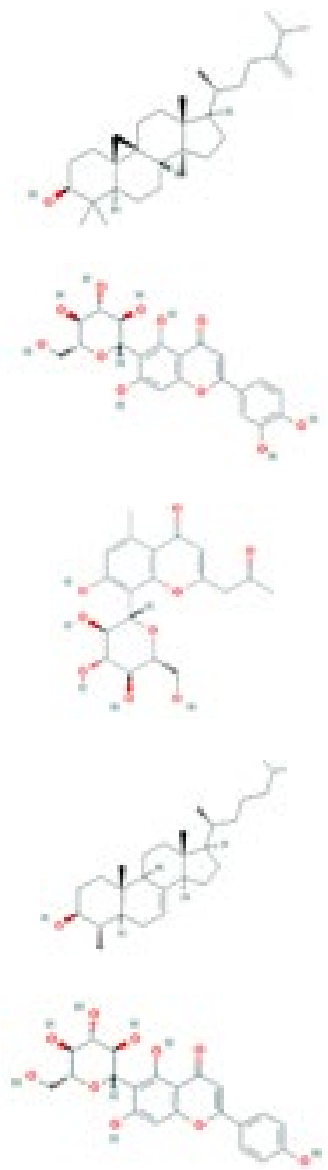
32

L-malic acid

33

Ethyl 2-oxo-3,4-dihydrochromene-3-carboxylate

34

Madagascine

35

Saponarin

36

Cinnamic acid

37

Fumaric acid

38

Ferulic acid

39

Sinapic acid

40

Caffeic acid
CID222656

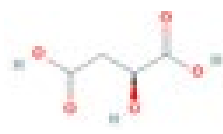

CID334443

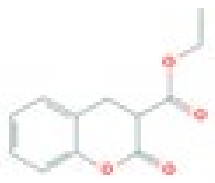

CID378693

CID441381

CID444539

CID444972

CID445858

CID637775

CID689043
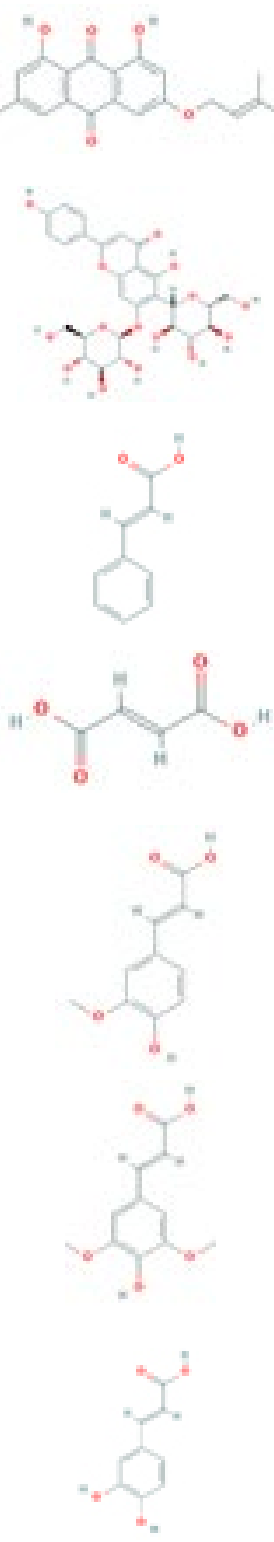
41 1-(2,4-Dihydroxy-6-methyl-

CID692802 phenyl)-2-(1-phenyl-1Hpyrazol-4-yl) ethan-1-one

42

cis-p-Coumaric acid

CID1549106

43

Chlorogenic acid

CID1794427

44

Aloesaponarin II

CID3085033

45

Quercetin

CID5280343

46

Apigenin

CID5280443

47

Luteolin

CID5280445

48

Quercitrin

CID5280459

49

Rutin

CID5280805
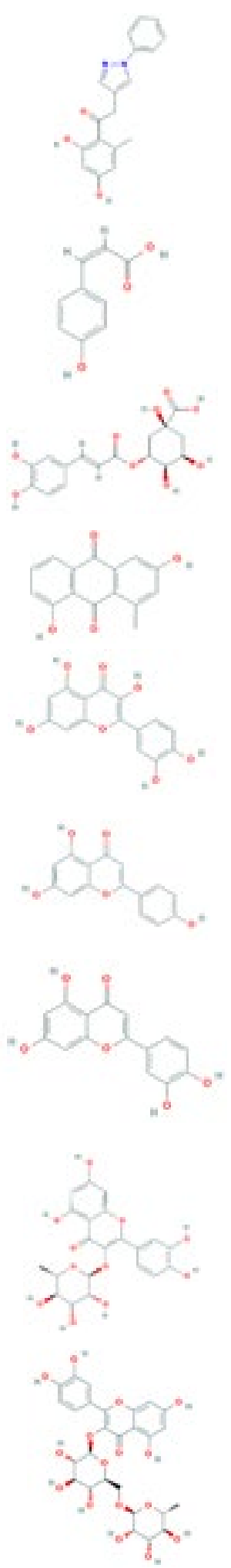
50

Kaempferol

Myricetin

51

52

53

Aloeresin A

54

7-Hydroxy-4-methoxy5-methylchromen-2-one

55

$$
\text { Geranyloxyemodin }
$$

56

(R)-3-(4-Hydroxyphenyl)-2(methylamino)propanoic acid

57

Methyl Aloesinyl cinnamate

58

Aloesaponarin

59

Barbaloin
CID5280863

CID5281672

CID5317333

CID5317657

CID5318268

CID5785070

CID7010357

CID9958939

CID11098986
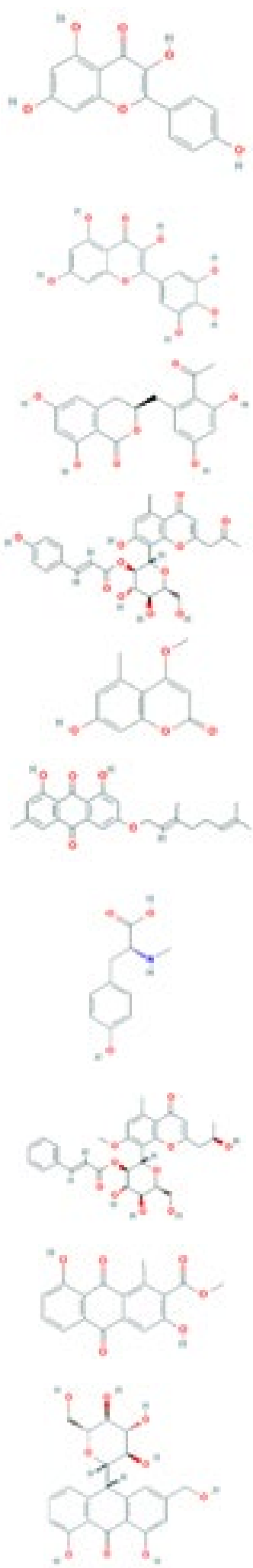
60

1,2,8-Trihydroxy-6-methylanthracene-9,10-dione

61

62

63

64

65

5-Feruloylquinic acid

66

Elgonica dimer A

67

Lutonarin
CID12313148

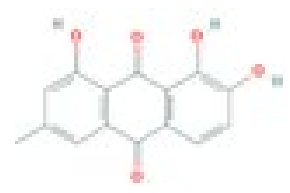

CID12760132

CID13984205

CID14211225

CID14729460
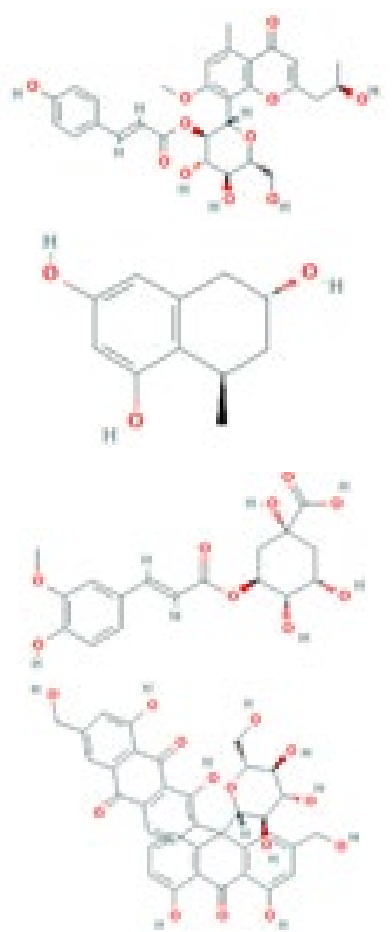

CID21582596

CID44257976
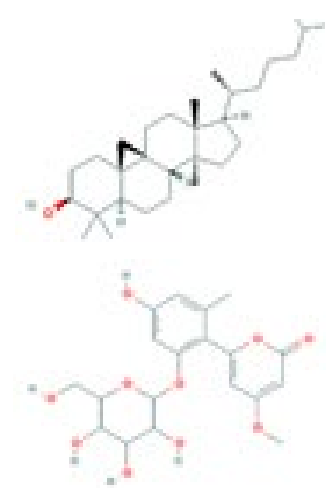

CID15901362

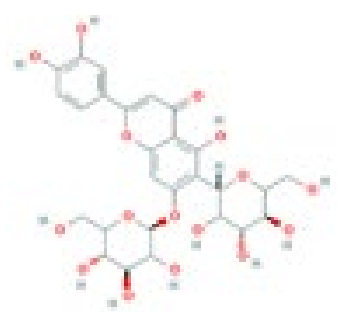


68 Aloinoside A

CID46173997

69

Aloinoside B

70 4-o-Caffeoylshikimic acid

71 Ascorbic acid

72 IsoAloeresin D 73 5-p-Coumaroylquinic acid

CID46173998

CID49821869

CID76332505
CID54670067

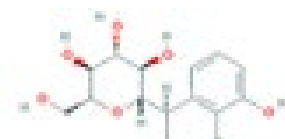

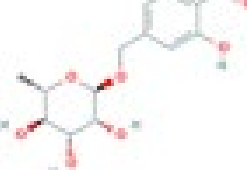

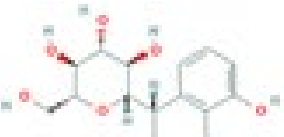<smiles>CC(C)=C1C(C)CC(CO[C@@H]2O[C@H](C)[C@@H](O)[C@H](C)[C@H]2O)C[C@H]1O</smiles><smiles>COc1ccc(C=CC(=O)OC2[C@H](O)CC(C(=O)O)C[C@H]2O)cc1O</smiles><smiles>O=C1O[C@H]([C@H](O)CO)C(O)=C1O</smiles>

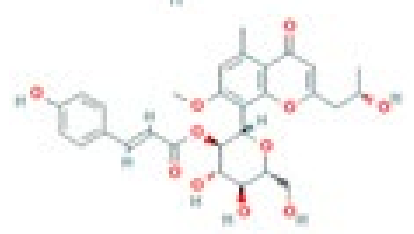

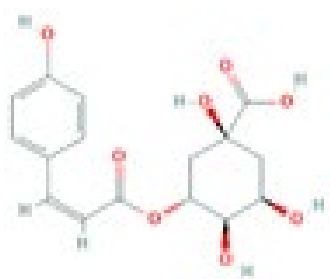


74 7-Hydroxyaloin A

75 Eremophilone lactone acid

76 Aloenin-2'-p-coumaroyl ester NeoAloesin A
CID102052685

CID102196083
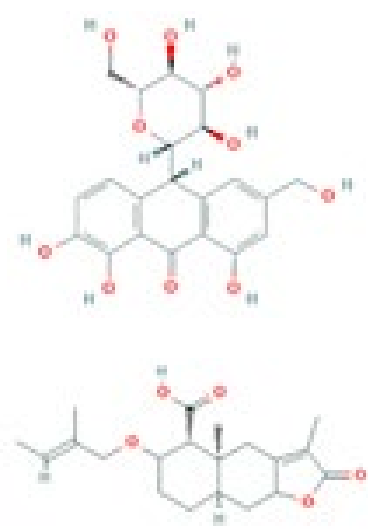

CID102435797

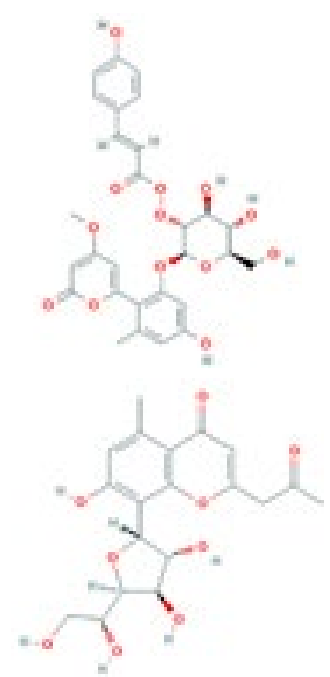

blood glucose levels. ${ }^{28}$ In addition, in other clinical trials, after taking whole leaf Aloe extract for 12 weeks in 60 patients with hyperlipidemia, serum total cholesterol, triglycerides, and LDL low-density lipoprotein levels were significantly reduced. ${ }^{28}$ Therefore, human clinical trial studies have provided promising preliminary data showing the significant beneficial effects of Aloe on diabetes and cardiovascular disease-related complications. Different studies have also been conducted in animal models using Aloe preparations, and these studies have shown that the effects on glucose and blood lipids are not consistent. In rodent models, two experiments were performed. First, Aloe latex was applied to alloxan-induced diabetic mice, ${ }^{29}$ and second, Aloe gel was applied to streptozotocin (STZ) induced diabetic rats. ${ }^{30}$ fasting blood glucose. Nevertheless, Aloe gel has been reported to increase glucose levels in tetraoxaneinduced diabetic rats. ${ }^{31}$ The anti-diabetic effect of processed Aloe gel was also studied in diet-induced obesity (DIO) mice, which is an animal model with metabolic abnormalities that are very similar to non-insulin-dependent human DM, including hyperglycemia, obesity, and insulin resistance. ${ }^{32}$

Similarly, Aloe gel extract has been found to reduce plasma insulin levels in STZ-induced diabetic rats and reduce the concentration of cholesterol, triglycerides, and free fatty acids in plasma, liver, and kidneys. ${ }^{33}$ The focus of this work is to identify the active ingredients of Aloe to prevent diabetes. Preliminary evidence shows that it has anti-diabetic properties and no side effects. 


\section{Structure and chemical constituents} Aloe vera for the treatment of Diabetes Milletus

Uptake of Aloe juice has been shown to improve blood glucose levels and lower blood pressure, making it useful in the treatment of diabetes. ${ }^{34}$ Additionally, acute hepatitis disease swelling was reduced, and wound injuries healed faster. The presence of compounds such as lectins, mannans and anthraquinones are responsible for various biological activities. ${ }^{35}$ Aloe juice has been acquiring ubiquity, and you may have seen the juice on store racks. However, what precisely right? What's more, is it bravo? There are various implied medical advantages that have been twirling around online media, one of which that it's useful for individuals with diabetes. Here's a gander at the science that looks at the impacts of Aloe juice on glucose in individuals with diabetes. It fills in environments that hot and dry, including the Mediterranean, Center East, India, and Africa. In the U.S., Aloe is developed in Florida, Texas, and Arizona. The plant is a delicious and its leaves are comprised of an unmistakable gel that helps the plant store water. You might be acquainted with Aloe gel, as it is normal used to mitigate burn from the sun. Aloe juice is produced using this equivalent gel. It's a thick, gooey fluid that is made by squashing or pounding the leaf of the Aloe plant to remove the gel, sifting it, and adding water to transform it into a fluid.

There is a modest quantity of examination that ganders at whether Aloe supplementation can be useful in overseeing diabetes. For instance, a 2016 deliberate audit and meta-examination took a gander at the impact of Aloe supplementation on glycemic control of individuals with prediabetes and type 2 diabetes. Eight preliminaries with an aggregate of 470 subjects were incorporated. The outcomes from these preliminaries propose that Aloe may affect blood glucose in individuals with prediabetes: the preliminaries found that Aloeal together further developed fasting blood glucose, however had no drawn-out impact on the hemoglobin A1C, which gives an image of blood glucose levels from the past 90 days. Specialists reasoned that in people with type 2 diabetes, Aloe may assist with further developing blood glucose control with a little improvement found in fasting blood glucose and a critical improvement in hemoglobin A1C. Albeit this meta-examination showed conceivable possible advantages of Aloe to assist with further developing glucose control in individuals with prediabetes and type 2 diabetes, they utilized various portions of Aloe supplements, not Aloe juice. A superior controlled randomized preliminary is expected to check whether there are any advantages of utilizing Aloe juice or standard measures of Aloe supplements.

In a 2015 randomized control preliminary, 72 individuals with side effects of prediabetes took Aloe cases (300 mg, $500 \mathrm{mg}$ treatment). The individuals who took Aloe cases showed a reduction in either fasting glucose or hemoglobin A1C following a month, or a decline in fatty oils (blood fat levels) following two months, contingent upon the dose they took. Analysts inferred that taking Aloe enhancements might actually assist with blood glucose the board in individuals with diabetes inside about a month and even assistance with blood lipid levels following two months. Once more, this examination was finished with supplements and not Aloe juice and with few members. Albeit the above research exhibits constructive outcomes found by utilizing Aloe supplements, the sorts of enhancements that were utilized in these examinations are not reliable. They incorporate different types of Aloe vera, for example, gel powder; separate, crude squashed leaves, and newly removed fluid. The dosages additionally changed fundamentally, as did the span of the investigations. Due to the variety between the investigations, the little example sizes, and the wide inconstancy of the structure and portion, the exploration truly doesn't hold a lot of legitimacy right now. Despite the fact that individuals given Aloe gel had further developed insulin resistance and weight reduction in comparison to the placebo group. The Aloe juice sold in business sectors will in general have added sugar, which won't assist with blood glucose the board and can really make it spike also, the proof with respect to Aloe all alone is restricted and not definitive as of now. It's ideal to avoid the Aloe juice to assist with dealing with your glucose. All things considered, attempt these three choices that examination shows can help. High glucose can damage organs if left untreated. A total of 382 million people in the world have diabetes, 
including type II diabetes. Compared with nondiabetic patients, diabetic patients are more likely to seek complementary and alternative medicines. How Aloe vera can benefit type 1 and type 2 diabetes: Study

The failure of your body to manage insulin causes diabetes (high blood sugar or glucose). Diabetes increases the chance of cardiovascular failure or stroke, as well as nerve damage, circulation difficulties, mild repairs, eyesight impairments, and contamination, according to the Mayo Clinic website. The Aloe plant has been used to treat wounds and consumption for thousands of years, and some data suggests that it can help reduce glucose levels. If you want to try Aloe to help with diabetes symptoms, your PCP can tell you if it's a good fit for this therapeutic intervention. Diabetes has several different types. Diabetic type 1 (T1D): Type 1 diabetes arises when the patient's body is unable to manufacture enough insulin and is fully reliant on insulin infusions. Type 2 diabetes is more common in adults and arises when the patient's cells are unable to use the insulin produced by the body adequately.

\section{How does diabetes affect?}

It has previously been reported that the absorption of Aloe juice helps to further increase blood sugar levels, which may subsequently be valuable for treating diabetic patients. In addition, Aloe is also related to: Reduce the lipid (fat) content of the patient's blood. The content of these particles is surprisingly significant. In the blood (for example, some people with type 2 diabetes) or potentially severe hepatitis (liver infection), growth slows and wound repair speeds up. Leg wounds and ulcers are normal misunderstandings of diabetes, and usually require more effort than healthy non-diabetic people to recover. This also indicates that cholesterol levels are lowered and total cholesterol is slightly improved. Several clinical trials have been released, revealing the anti-diabetic properties of Aloe vera. Diabetic patients can also get obvious help from Aloe blood enhancement. Because Aloe can reverse "blood oozing" and elevate transmission to the furthest point, diabetic patients with limbic neuropathy (numbness in the hands and feet) can get significant help from Aloe supplements Diabetics feel better with Aloe juice. Regular extraction of
Aloe into the container structure found that Aloe was fundamentally superior to sham treatment in lowering glucose levels and damaging lowdensity lipoprotein cholesterol, without specifying harmful effects. Blue Safeguard Integral and Elective Wellbeing agrees that Aloe has been shown to reduce glucose and cholesterol levels in animal and human studies, and recorded typical measurements of 5 to 15 milliliters of Aloe juice, which requires twice as much per day. Aloe is rich in strong plant compounds that can relieve pain and expand and promote healing. Polysaccharides, including a substance called acetylmannan, as a conventional analgesic and therapeutic agent, can also further promote blood flow and oxygenation. Allantoin is a characteristic compound found in Aloe vera. It is sticky, which means it has a soothing and analgesic effect.

What exactly is the link connecting Aloe vera and diabetes?

Diabetes may be the best-known infection affecting the general population today. This situation is very problematic for health professionals, because the number of hyperglycemic individuals is increasing and we have not changed our lifestyle. Considering the dangers of diseases like diabetes, health experts recommend that you improve your lifestyle, especially yours eating habits. The plant's gel controls the glucose in the circulatory system and improves the personal satisfaction of diabetic patients. Normal use of Aloe can promote the health of the kidneys and colon, which are usually affected by diabetes. Combining Aloe with a daily low-starch diet can help relieve diabetes naturally. Aloe contains glucomannan, which is essential for lowering glucose levels due to its soluble fiber content. Hemicellulose can also completely lower glucose. Anthraquinones, natural phenolic compound, and lectins are all important components of Aloe gel for keeping blood sugar levels low. Aloe can lower your blood sugar in half in just two months. Aloe can aid in the outflow of toxins and waste from the body, as well as lowering the amount of sugar in the circulatory system. Regular usage of Aloe to cure diabetes can assist with various issues such as ulcers, wounds, and infections, so there are no limitations due to its strong oxidant content. 


\section{How should you incorporate Aloevera into your diet?}

To reduce your glucose levels, try consuming pure Aloe gel produced directly from plants. Our Eternity Aloe gel is made entirely of Aloe vera. It's similar to slicing an Aloe leaf and ingesting the gel directly from the plant. Aloe gel with a regulated $\mathrm{pH}$. During the processing, no synthetic ingredients were used. Use only standard pure binding. 12 vitamins (B1, B2, B3, B6, B12, C, E), 18 amino acids, and 20 minerals are found in Aloe leaves.

\section{CONCLUSION}

Diabetes is a significant public health issue. The global diabetes epidemic has had a tremendous impact on India, and the disease burden has increased dramatically. Diabetes is quickly increasing in prevalence, especially in Indian cities, according to data. As a result, diabetes-related complications are also increasing, significantly increasing overall morbidity and mortality. The country's low level of education and lack of awareness about the disease are increasing its impact on the health of the population. Early identification and treatment at the grassroots level can reduce morbidity and mortality. Drug therapy is the cornerstone of diabetes management, when combined with non-drug therapy, diabetes is now basically controllable. Although there are currently a variety of medications available, none of them have no side effects. The tolerability and safety of overdose of many antidiabetic drugs is also an issue. Many effective medications come from plant sources. In this review, we should pay attention to Aloe vera, that with the development of traditional medicine, Aloe gel can be used to treat various diseases. Some reports have questioned the safety and efficacy of Aloe vera or its compounds, especially at different doses, and some studies have shown no side effects. Since Aloe vera is part of the daily diet in many Asian countries and appears to be non-toxic, it is necessary to investigate whether Aloe vera dietary supplement can be a beneficial preventive or nutritional mitigation strategy to reduce the effects of diabetes.

\section{ACKNOWLEDGMENTS} None.

\section{CONFLICT OF INTEREST}

The authors declare that there is no conflict of interest.

\section{AUTHORS' CONTRIBUTION}

All authors listed have made a substantial, direct and intellectual contribution to the work, and approved it for publication.

\section{FUNDING}

None.

\section{DATA AVAILABILITY}

All datasets generated or analyzed during this study are included in the manuscript.

\section{ETHICS STATEMENT}

Not applicable.

\section{REFERENCES}

1. Nathan DM, Genuth S, Lachin J, et al. The effect of intensive treatment of diabetes on the development and progression of long-term complications in insulin-dependent diabetes mellitus. N Eng J Med. 1993;329(14):977-986. doi: 10.1056/ NEJM199309303291401

2. American Diabetes Association. Economic costs of diabetes in the US in 2012. Diabetes care. 2013;36(6):1797. doi: 10.2337/dc13-er06

3. Hood KK, Peterson CM, Rohan JM, Drotar D. Association between adherence and glycemic control in pediatric type 1 diabetes: a meta-analysis. Pediatrics. 2009; 124(6):e1171-e1179. doi: 10.1542/peds.2009-0207

4. Morris AD, Boyle DI, McMahon AD, et al. Adherence to insulin treatment, glycaemic control, and ketoacidosis in insulin-dependent diabetes mellitus. The Lancet. 1997;350(9090):1505-1510. doi: 10.1016/S01406736(97)06234-X

5. Amiel SA, Sherwin RS, Simonson DC, Lauritano AA, Tamborlane WV. Impaired insulin action in puberty. A contributing factor to poor glycemic control in adolescents with diabetes. $N$ Engl J Med. 1986;315(4):215-219. doi: 10.1056/ NEJM198607243150402

6. Svoren BM, Volkening LK, Butler DA, Moreland EC, Anderson BJ, Laffel LM. Temporal trends in the treatment of pediatric type 1 diabetes and impact on acute outcomes. J Pediatr. 2007;150(3):279-285. doi: 10.1016/j.jpeds.2006.12.009

7. Silverstein J, Klingensmith G, Copeland K, et al. Care of children and adolescents with type 1 diabetes: a statement of the American Diabetes Association. Diabetes Care. 2005;28(1):186-212. doi: 10.2337/ diacare.28.1.186

8. Unnikrishnan AG, Bhatia E, Bhatia V, et al. Type 1 diabetes versus type 2 diabetes with onset in persons younger than 20 years of age: results from an Indian 
multicenter study. Ann N Y Acad Sci. 2008;1150(1):239244. doi: 10.1196/annals.1447.056

9. Bai PA, Krishnaswami CV, Chellamariappan M, Kumar GV, Subramaniam JR. Glycosuria and diabetes mellitus in children and adolescents in south India. Diabetes Res Clin Pract. 1991;13(1-2):131-135. doi: 10.1016/01688227(91)90043-D

10. Staines A, Hanif S, Ahmed S, McKinney PA, Shera $\mathrm{S}$, Bodansky HJ. Incidence of insulin dependent diabetes mellitus in Karachi, Pakistan. Arch Dis Child. 1997;76(2):121-123. doi: 10.1136/adc.76.2.121

11. Raymond NT, Jones JR, Swift PG, et al. Comparative incidence of Type I diabetes in children aged under 15 years from South Asian and White or Other ethnic backgrounds in Leicestershire, UK, 1989 to 1998. Diabetologia. 2001;44(3):32-36. doi: 10.1007/ PL00002951

12. Harron KL, McKinney PA, Feltbower RG, et al. Incidence rate trends in childhood Type 1 diabetes in Yorkshire, UK 1978-2007: effects of deprivation and age at diagnosis in the south Asian and non-south Asian populations. Dia Med. 2011;28(12):1508-1513. doi: 10.1111/j.1464-5491.2011.03413.x

13. Noble JA, Erlich HA. Genetics of type 1 diabetes. Cold Spring Harb Perspect Med. 2012;2(1):a007732. doi: 10.1101/cshperspect.a007732

14. Tattersall RB, Pyke DA. Diabetes in identical twins. The Lancet. 1972;300(7787):1120-1125. doi: 10.1016/ S0140-6736(72)92720-1

15. Das AK. Type 1 diabetes in India: Overall insights. Indian J Endocrinol Metab. 2015;19(Suppl 1):S31-S33. doi: 10.4103/2230-8210.155372

16. Moradi B, Abbaszadeh S, Shahsavari S, Alizadeh M, Beyranvand $F$. The most useful medicinal herbs to treat diabetes. Biomed Res Ther. 2018;5(8):2538-2551. doi: 10.15419/bmrat.v5i8.463

17. Kooti W, Farokhipour M, Asadzadeh Z, Ashtary-Larky $D$, Asadi-Samani M. The role of medicinal plants in the treatment of diabetes: a systematic review. Electronic Physician. 2016;8(1):1832. doi: 10.19082/1832

18. Aloe Vera and Diabetes. 2019. https://www.diabetes. co.uk/natural-therapies/aloe-vera.html

19. Dagne E, Bisrat D, Viljoen A, Van Wyk BE. Chemistry of Aloe species. Curr Org Chem. 2000;4(10):1055-1078. doi: 10.2174/1385272003375932

20. Boudreau MD, Beland FA. An evaluation of the biological and toxicological properties of Aloe barbadensis (miller), Aloe vera. J Environ Sci Heal C. 2006;24(1):103154. doi: 10.1080/10590500600614303

21. Patel K, Tahilyani V. Barbaloin: a concise report of its pharmacological and analytical aspects. Asian Pac J Trop Biomed. 2012;2(10):835-838. doi: 10.1016/ S2221-1691(12)60239-1

22. Mortensen A. Carotenoids and other pigments as natural colorants. Pure Appl Chem. 2006;78(8):14771491. doi: $10.1351 /$ pac200678081477

23. Hamman. World Health Organization. WHO Monographs on Selected Medicinal Plants. Vol. 1.
Geneva: World Health Organization. 1999. https:// apps.who.int/iris/handle/10665/42052

24. Radha MH, Laxmipriya NP. Evaluation of biological properties and clinical effectiveness of Aloe vera: A systematic review. J Tradit Complement Med. 2015;5(1):21-26. doi: 10.1016/j.jtcme.2014.10.006

25. Coronado GD, Thompson B, Tejeda S, Godina R. Attitudes and beliefs among Mexican Americans about type 2 diabetes. $J$ Health Care Poor Underserved. 2004;15(4):576-588. doi: 10.1353/hpu.2004.0057

26. Yeh GY, Eisenberg DM, Kaptchuk TJ, Phillips RS. Systematic review of herbs and dietary supplements for glycemic control in diabetes. Diabetes Care. 2003;26(4):1277-1294. doi: 10.2337/diacare.26.4.1277

27. Feinberg $S$, Williams $R$, Hagler GS, et al. Long-term evaluation of air sensor technology under ambient conditions in Denver, Colorado. Atmos Meas Tech. 2018;11(8):4605-4615. doi: 10.5194/amt-11-46052018

28. Foster M, Hunter D, Samman S. Evaluation of the nutritional and metabolic effects of Aloe vera. Herbal Medicine: Biomolecular and Clinical Aspects. 2nd ed. Boca Raton:Benzie and Wachtel-Galor; 2011. doi: 10.1201/b10787-4

29. Sanchez M, Gonzalez-Burgos E, Iglesias I, GomezSerranillos MP. Pharmacological update properties of Aloe vera and its major active constituents. Molecules. 2020; 25(6):1324. doi: 10.3390/molecules25061324

30. Rajasekaran NS, Nithya M, Rose C, Chandra TS. The effect of finger millet feeding on the early responses during the process of wound healing in diabetic rats. Biochim Biophys Acta. 2004;1689(3):190-201. doi: 10.1016/j.bbadis.2004.03.004

31. Atanu FO, Avwioroko OJ, Momoh S. Anti-diabetic effect of combined treatment with Aloe vera gel and Metformin on alloxan-induced diabetic rats. J Ayu Her Integr Med. 2018;4(1):1-5. doi: 10.31254/ jahm.2018.4101

32. Kim K, Kim H, Kwon J, et al. Hypoglycemic and hypolipidemic effects of processed Aloe vera gel in a mouse model of non-insulin-dependent diabetes mellitus. Phytomedicine. 2009;16(9):856-863. doi: 10.1016/j.phymed.2009.02.014

33. Rajasekaran S, Ravi K, Sivagnanam K, Subramanian S. Beneficial effects of Aloe vera leaf gel extract on lipid profile status in rats with streptozotocin diabetes. Clin Exp Pharmacol Physiol. 2006;33(3):232-327. doi: 10.1111/j.1440-1681.2006.04351.x

34. The Effect of Aloe Vera on Diabetes - Benefits, Drawbacks, How to Take. 2020. https:// prescriptionhope.com/blog-the-effect-of-aloe-veraon-diabetes/

35. Yongchaiyudha S, Rungpitarangsi V, Bunyapraphatsara $\mathrm{N}$, Chokechaijaroenporn O. Antidiabetic activity of Aloe vera $\mathrm{L}$. juice. I. Clinical trial in new cases of diabetes mellitus. Phytomedicine. 1996;3(3):241-243. doi: 10.1016/S0944-7113(96)80060-2 